2017-07-01

\title{
Human-Robot Interaction and
}

\section{Neuroprosthetics: A review of new technologies}

\section{Cangelosi, A}

http://hdl.handle.net/10026.1/9872

\subsection{9/MCE.2016.2614423 \\ IEEE Consumer Electronics Magazine}

All content in PEARL is protected by copyright law. Author manuscripts are made available in accordance with publisher policies. Please cite only the published version using the details provided on the item record or document. In the absence of an open licence (e.g. Creative Commons), permissions for further reuse of content should be sought from the publisher or author. 


\title{
New Technologies for Human Robot Interaction and Neuroprosthetics
}

\author{
Angelo Cangelosi, Sara Invitto
}

\begin{abstract}
New technologies in the field of neuroprosthetics and robotics are leading to the development of innovative commercial products based on user-centered, functional processes of cognitive neuroscience and perceptron studies. The aim of this review is to analyze this innovative path through the description of some of the latest neuroprosthetics and human-robot interaction applications, in particular the Brain Computer Interface linked to haptic systems, interactive robotics and autonomous systems. These issues will be addressed by analyzing developmental robotics and examples of neurorobotics research. These new devices show the benefits of using an interdisciplinary approach based on cognitive neuroscience, embodied and situated cognition and neural network and deep learning. All these products share the capability to adapt, on-line, to the dynamic environment and to the user's actions. As the role of the human tutor is key in this learning process, these systems permit a natural and dynamic approach to interaction between people, neuroprosthetics and robotics selfextensions.
\end{abstract}

Index Terms - Intelligent Robots, Neurocontroller, Neurofeedback, Neuromorphics, Neuroprosthetic

\section{INTRODUCTION}

$\mathrm{T}$ he use of cognitive and computational neuroscience has applications in the field of consumer electronics for the production of elements and instrumentation for both interactive robotic interfaces and diagnostic systems. Recent research on the neuroprosthetics market suggests that the business in these branches will experience considerable development in the coming years[1]. Neuroprosthetics includes developing technologies such as Deep Brain Stimulation, Vagus Nerve Stimulation, Spinal Cord Stimulation and others to stimulate motors, visual, auditory, haptic perception and cognitive processing: for example, the construction of neuromorphic elements (e.g., haptic effectors that have been built in recent years), which are used in the Brain-Computer Interface (BCI) both for recreational and clinical / therapeutic purposes.

A. Cangelosi is Director of the Center for Robotics and Neural Systems, CNRS, University of Plymouth, UK (e-mail: A.Cangelosi@plymouth.ac.uk). $\mathrm{S}$. Invitto is assistant professor of General Psychology, Department of Biological and Environmental Sciences and Technologies, University of
These developments in neuroprosthetics are closely linked to the recent significant investment and progress in research on neural networks and deep learning approaches to robotics and autonomous systems [2][3]. Specifically, one key area of development has been that of cognitive robots for human-robot interaction and assistive robotics. This concerns the design of robot companions for the elderly, social robots for children with disabilities such as Autism Spectrum Disorders, and robot tutors for school and education[4][5][6]. Other areas of application focus on joint-action, that is, collaborative tasks where a human and a robot share workspace for joint object manipulation as in assembly tasks.

The research into social and collaborative robots has required a shift in approach to robot design: from robot pre-programming to robot learning. State of the art of commercial these systems, as in manufacturing and assembly robots, imply the precise preprogramming of the robot's actions and the safe separation of

Text the robot workspace from human one. However, as robot companions are required to share their environment with that of human users, it is essential to design robots who can dynamically and safely adapt their behaviour to that of people, to avoid any harm to human users. Moreover, as shared human spaces are dynamically changing and unpredictable environments, robots have to be able to adapt and learn how to cope with changing situations and with individual users' specific needs and preferences.

In the following sections, we will review some of the latest developments in the fields of neuroprosthetics and BCI (section II), in cognitive and neurorobotics systems for human-robot interaction (Section III) and in robotics and neuroprosthetics hybrid systems (IV). The first set of studies concerns the possibility to use a computer as a neural interface[7], this approach has developed devices that are also known as 'Brain Computer Interface'. These tools are part of the recent field of scientific neuroprosthetics application [8]. The second set of reviewed works focuses on developmental robotics approaches to human-machine interaction for embodied language learning tasks, and integrated neurorobotics and neuromorphic systems for autonomous robot behavior.

These applications show the advantages of developing a natural and dynamic approach to interaction between users and neuroprosthetics, robotic and hybrid systems

Salento and Institute of Nanoscience - CNR National Research Council U.O.S. Euromediterranean Center for Nanomaterial 


\section{NEUROPROSTHETICS AND BCI}

The field of neuroprosthetics started in the 1970s when Vidal [9] published a seminal article on the development of bioengineering and neuroscience, in which he described how cortical responses recorded by an electroencephalogram (with very low frequencies $<1 \mathrm{~Hz}$ up to $30 \mathrm{~Hz}$ ) could be interfaced to a digital system.

This was achieved specifically through the analysis of event related potentials (ERPs), responses evoked by sensory stimuli in certain attentional and perceptive tasks. In this model, the $\mathrm{BCI}$ - is a tool that interfaces with the particular EEG responses and transduces them through a digital interface. For example, if the system is able to frame signal patterns in the brain responses of lateralized movement or of a negative or positive response, the system can be interfaced to a digital system that responds through biofeedback.

The BCI system was, at first, especially useful for individuals with motor paralysis, amyotrophic lateral sclerosis, and in some cases coma (even interfacing responses such as saccadic eye movements and reflexes). Industry and academic neuroengineering research, applied to neurodegenerative diseases, has involved the development of EEG systems that can guarantee an acceptable degree of autonomy and communicative ability to people with severe disabilities.

There have been numerous studies, especially in the last decade, and each of these had the aim of capturing a new facet, or the latent potential of this system which, to date, still proves to have great growth potential for the imminent future. The evolution of techniques that provide a better cataloging of data and much more effective interactions has, however, led to increasing capacity for neural control and to the emergence of different branches of application within the BCI. For example, assistive $\mathrm{BCI}$ concerns the restoration of mobility, the ability to express oneself, and the ability to control one's environment.

$\mathrm{BCI}$ has been used before with respect to the most rudimentary form of communication (i.e., "yes" or "no" choices) using the technique of slow cortical potentials that, when adjusted to moving a cursor, employed the verbal dichotomy of assertiondenial (YES-NO). Subsequently the response action range has widened, allowing users to compose increasingly complex words.

This was possible thanks to the research that led to the protocol of the "Farwell-Donchin Matrix", which captures P300 ERPs component, evoked in response to the random flash of letters that are part of a matrix and links them to a specific letter [10]. As for the mobility and action in this context, there have been numerous efforts by the BCI research community to associate this device with existing tools in order to support them. Besides the commands function, with the addition of assistive-efficacy in the therapeutic purpose, BCI was developed for diagnostics and to provide an increase in cognitive functions (e.g. motor imagery experiments) (i.e. a flexible BCI system using dry electrodes fig.1).

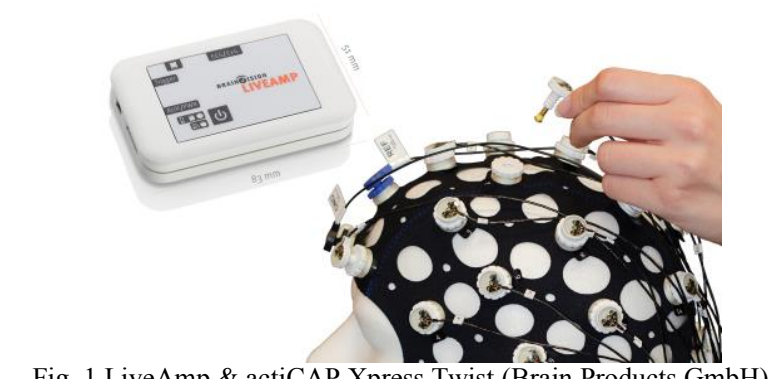

Fig. 1 LiveAmp \& actiCAP Xpress Twist (Brain Products GmbH).

Users can now monitor changes in EEG rhythms through the practice of meditation, e.g. classify images according to electroencephalographic responses to visual stimuli and monitor their alertness, which is closely linked to $\alpha$ rhythms (specific brain waves ranging from 7.5 to $12.5 \mathrm{~Hz}$ ). In a recent and innovative instrumentation by the g.tec company, an interface was linked to auditory biofeedback and vibro-tactile stimulators. The mindBEAGLE $\AA$ system[11] is a portable medical grade biosignal amplifier, with an EEG cap with active electrodes, a standard laptop computer with the mindBEAGLE®software, in-ear phones for auditory stimulation and vibro-tactile stimulators attached to the patient's body (fig.2). The development of the new mindBeagle software was supported by the European Union H2020 program, through the SME Inst project "ComAware".

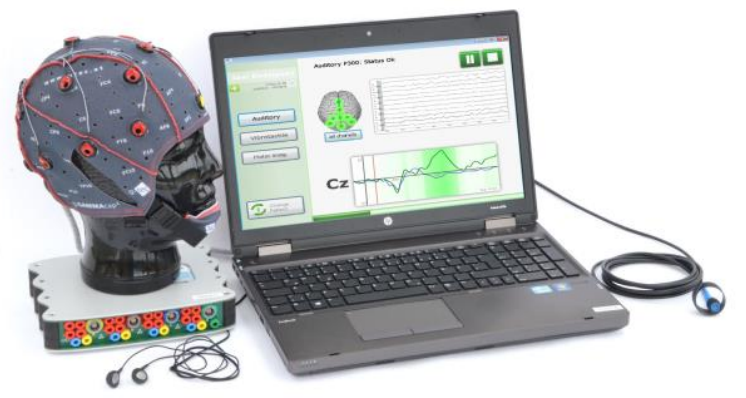

Fig. 2 MindBEAGLE®system (g.tec company).

More recently, BCI has taken the first steps in the field of entertainment, when simple guided gaming peripherals using the principle of artificial intelligence have been made available to a wider audience. The first system to implement the control of neuronal signals useful for computer games was the Berlin BCI [12] which, a little later, included cerebral versions of table tennis and PacMan. Afterwards, new BCIs were introduced to control the game, that were specific to the user's attention based on the degree of relaxation, and could be monitored by means of $\alpha$ rhythms of the frontal cortex. These were developed as serious games too. The evolution of these products has led to NeuroSky software[13], which is sold with a wearable EEG cap device that allows increasing attentional arousal during children's and adult's games[14]. Additional interfaces are emerging even within augmented and virtual reality games. The power to mentally represent a task and to mentally make a gesture, allows the user, in the case of virtual environments, to interact with the environment or other objects through a motor 
imagery or global perception system and motor action in the absence of haptic feedback[15]. With regard to music and the visual arts, however, the imagery factor alone is not enough to edit an interface; the BCI approaches involved in these fields require an effective level of patient preparation and constant training to enable users to memorize different kinds of stimuli. Haptic applications for commercial use have been developed in more recent times, and the state of the art with regard to these effectors is particularly innovative. A haptic interface is a device that allows us to maneuver a robot, real or virtual, and receive feedback in the form of tactile sensations. The user of a haptic interface is able to produce and use motor actions, such as physically manipulation of the interface, which in turn display and stimulate tactile kinaesthetics sensory information. Specifically, when participants interact with objects in the physical world, they implement intentional schemes enabling them to place and represent the effects of their actions. In the field of neuroscience, it is extremely important to develop or enable impaired haptic motor function or motor skills because the system also permits the integration of other cognitive systems. The sensorimotor grounding of the conceptual content shows how it could be involved in many more aspects of human cognitive life. For example, these aspects are strongly highlighted in Vittorio Gallese's "neural exploitation" hypothesis[16] and Susan Hurley's "shared circuits model"[17]. According these theories, cognition has a basis in sensorymotor integration and action.

Considering the hybrid bionic connection between the artifact and the nervous system, the proofs of feasibility of restoration of tactile capabilities have recently been provided in studies with human amputees. However, the restoration of fine tactile skills, such as the categorization of textural features, is still missing. One of the first animal models applied to the haptic system was a robot able to actively explore the surrounding environment with tactile sensors. This was developed by reproducing the biological details of the rat's whisker behavior from the modeling and physical implementation of the whisker [18] primary afferent neurons, and midbrain tactile information processing. Later, devices were developed further, including one developed by the Disney research group, that developed REVEL [19], a tactile system technology for augmented reality. This is a very interesting approach that mixes haptic stimulation and augmented manipulation. In this device, the user feels the haptic texture of a real object while observing it in an augmented reality display. This interaction can be integrated in a game, but it could also be interesting to extend it in a cognitive and perceptive task in serious games too. So, this field of research can be easily adapted for entertainment and brain training applications.

As for the link of haptic prosthetics and robotics, according to Gerald Loeb, a co-founder of SynTouch, and co-inventor of the BIOTAC technology (among many other patents covering diverse areas of engineering and neural prosthetics), one of the most relevant problems in neuroprosthetic is not having an haptic/tactile feedback on a robotic arm[20][21]. BIOTAC (fig.3) is a sensor able to perceive force, vibration and temperatures and to act as a haptic extension of a damaged or non-existent limb.

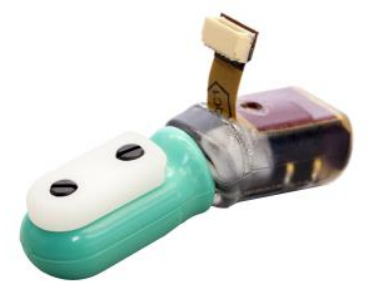

Fig. 3 BIOTAC (Syn Touch) sensor. BIOTAC is a sensor able to perceive force, vibration and temperatures and to act as a haptic extension of a damaged or non-existent limb.

Numerous other works are emerging in the haptic field. One of the most recent and interesting approaches is presented in a paper from the BioRobotic Institute of Pisa[22], that proposes a neuromorphic sensor capable of making sense of various grains / textures. This neuromorphic stimuli system is able to encode naturalistic textures under different sensing conditions and might therefore be suited for tactile information processing in real-life applications.

Agashe et al. [23] in which they connected a BCI system to noninvasive neuroprosthetic device. In this case, the prosthesis predicts the shape of the hand during grasping through brain signals. The cerebral mu-rhythms (specific brain waves ranging from 10 to $22 \mathrm{~Hz}$ ) are taken as the control indicator of the subject in locomotor stimulation. These particular brain waves are the equivalent of alpha waves recorded for the visual system, but are located in the motor cortex. They are a synchronous pattern of cortical electrical activity that is desynchronized during subject movement. Finally, Pfurtscheller [24] describes 'the hybrid BCI' that can process in parallel two different EEG conditions: Event Related Desynchronization/Synchronization (ERD/ERS) and steadystate visual evoked potentials (SSVEP).

\section{COGNITIVE AND NEUROROBOTICS MODELS FOR HUMAN- ROBOT INTERACTION}

These important developments in BCI and prosthetics for hybrid human-machine systems have been complemented by advances in cognitive and neuro-robotics and in human-robot interaction. Cognitive robotics [25] offers a suitable approach to the design of robots capable of learning and collaborating with humans. Cognitive robotics (also known as cognitive systems approach) concerns the design of robotic agents that are capable of learning from interaction with humans and with a sensorimotor control system directly inspired by the principles and mechanisms of behaviour control observed in natural (animals and humans) cognitive systems. Examples of cognitive robotics approaches include evolutionary robotics [26], based on the use of evolutionary computation methods to evolve the robot's controllers during their interaction with their physical and social environment, developmental robotics [27] which models the gradual acquisition of behavioral and cognitive capabilities in robots following child psychology principles and mechanisms, and neurorobotics [28], for the use 
of artificial neural networks (and brain-machine interfaces) for robot control and learning.

All these approaches share four key principles in the design of social robots interacting with people: (i) embodiment, i.e. the robot's sensors and actuators shape the type of behaviour and cognitive control strategy developed by the robot [29][30]; (ii) situatedness, i.e. where cognition is situated in the agent's interaction history in its physical and social environment [31]; (iii) grounding, where the agent's internal representations, in regard to language, are directly and intrinsically grounded in its experience of the world [32][33]; (iv) learning, as the agent's behavioural and cognitive strategies are autonomously acquired during its lifetime through evolutionary and/or developmental learning, for example implemented with neural networks, reinforcement learning or other machine learning techniques.

In the next section, we will look at two examples of cognitive robotics applications exploiting the four key principles. The first approach shows the advantages of using a developmental robotics approach which implements the four design principles: this consist of an experiment where the robot uses its body posture (embodiment principle) to acquire language via situated human-robot interaction (situatedness principle) to ground the language (grounding principle) in the robot's task and representation. Language is acquired (learning principle) during development. The second example focuses on neurorobotics, with a specific emphasis on the implementation for neural network controllers in neuromorphic systems. This puts the main emphasis on the learning principle, though within a situated and embodied interaction with its world.

\section{A. Interacting with robots: A developmental language learning approach}

Developmental Robotics is the "interdisciplinary approach to the autonomous design of behavioral and cognitive capabilities in artificial agents (robots) that takes direct inspiration from the developmental principles and mechanisms observed in natural cognitive systems (children)" [27]. As such this approach puts a strong emphasis on embodied and situated interaction between the (baby) robot and its caregiver or human tutor, taking inspiration from child development. For example, recent developmental psychology studies have investigated how body posture might be playing a critical role in early word learning [34]. To learn anything at all from real time experiences, a physical learner must be able to orient its sensors, and thereby its physical body, in order to attend to the referred object. Part of the learning challenge then is to react appropriately, e.g. orienting to the spatial locations of objects. Here we briefly present an embodied developmental approach, mapping the body posture to expected sensory experience for the learning of object names.

Morse et al. [35] use the humanoid robot iCub specifically to show the role of embodiment and body posture in supporting early word learning both in human infants and in baby robots. The robot's control system uses the Epigenetic Robotics Architecture (ERA), which is a connectionist model combining self-organising maps (for the robot's categorization abilities) and Hebbian learning (for the learning of object-name associations) (fig. 4a). To highlight the relevance of this approach, we show a short example using epigenetic [36] architecture as a metaphor in the construction of learning systems. Using this paradigm, we can compare the epigenetic approach to both robotics and neuroprosthetics learning processing. The term epigenetics, originally used by Jean Piaget, emphasizes the role of both the environment and of genetics in development. The ERA robotics example also refers to the concept of a Hebbian Network [37]: a simulation based on a concept closely connected to epigenetics, where the 'connective model' becomes predominant, expressing itself to build simulations of 'brain plasticity' and connections learned in an epigenetic way. So, a set of Kohonen self-organized maps [38] are pre-trained for object classification according to their color and shape (color and space maps). These maps tend to data reduction, with the consequence of a specialization of neural networks, rather than to its expansion. This neural model increases the connectivity of the system by reducing the number of artificial connections (nodes)In this case, the network models are based on a competitive learning algorithm. In addition, in ERA an extra Kohonen map is pre-trained to recognize the robot's body posture (posture map). A set of Hebbian associative learning weights, connecting the nodes between different Kohonen maps, are trained during language training sessions with a human teacher. These are directly inspired by Samuelson et al. [34] child psychology studies. During these language learning sessions (fig. 4b), the iCub changes its own posture to attend to different parts of the scene (i.e. to the left and the right side of the robot's peri-personal space) where two objects are shown. The Hebbian weights are then adjusted to create new associations between the visual color/shape maps, the posture map, and the nodes representing words. This is the key mechanism implementing language grounding in perception and action. The same modeling setup has also been used not only to replicate previous child psychology experiments, but also to make predictions on additional phenomena. This for example includes the prediction on the role of body posture in reducing interference between two competing cognitive tasks (later demonstrated in new child experiments - see data reported in [35]). Moreover, the ERA developmental robotics architecture has been extended to new robotic experiments on the role of embodiment in the acquisition of complex, abstract concepts [39], and on the role of finger counting and pointing gestures in number learning [40].

These developmental robotics experiments and architecture show the benefits of using an embodied and situated approach to learning. The developmental learning architecture endows robots with the capability to adapt, on-line, to the dynamic environment it is experiencing. As the role of the human tutor is key in this learning process, this permits a natural and dynamic approach to interaction between people and social robot companions. 

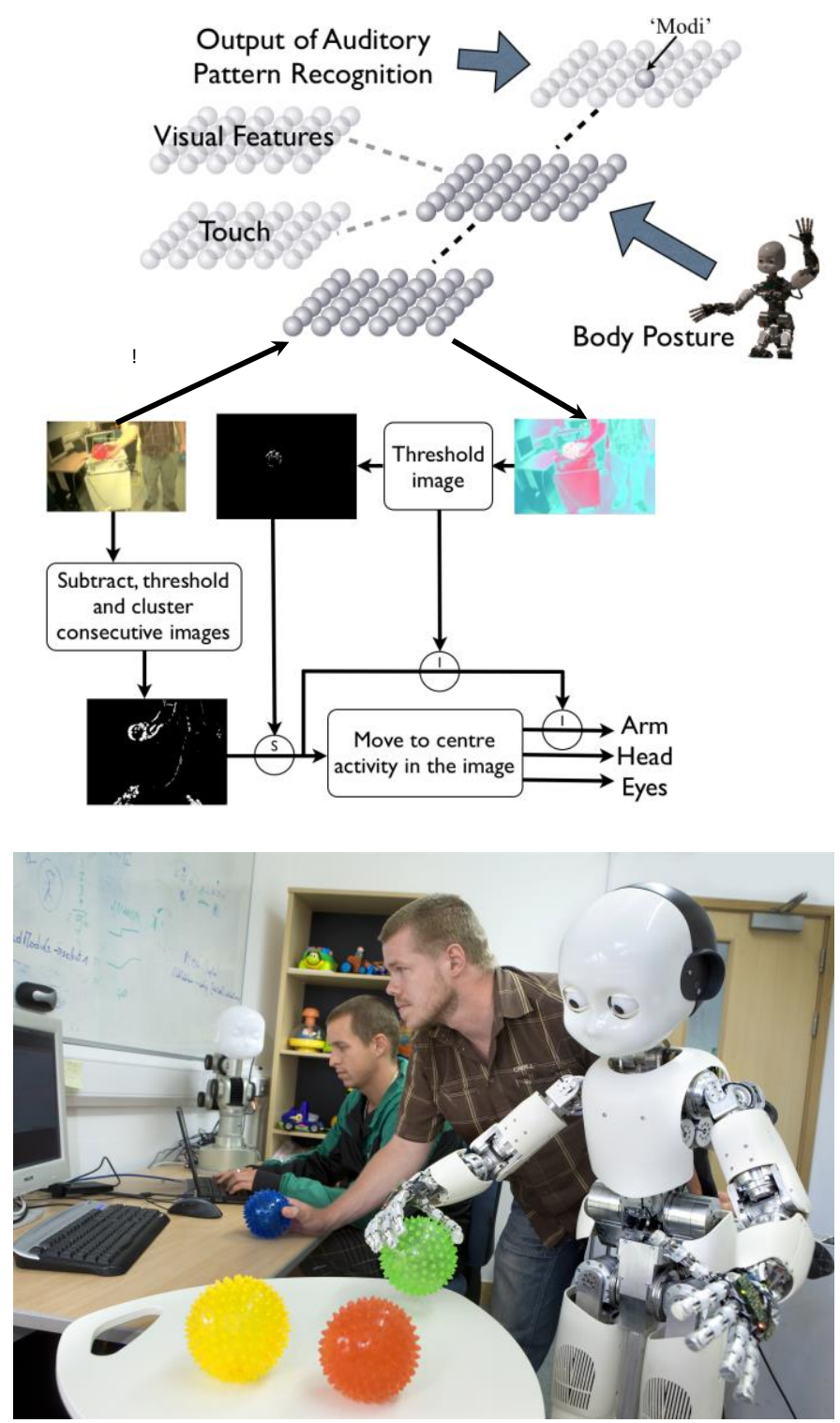

Fig. 4: (a, top) Cognitive architecture for the developmental language learning experiments. The top layers correspond to Kohonen maps connected by Hebbian learning weighs, whilst the blocks below show image processing processes. (b, below) Language learning experimental setup.

\section{B. Robots and neuromorphic systems: A neurorobotics model of attention}

Neurorobotics approaches put great emphasis on learning, as they use control architecture based on artificial neural networks and on sub-symbolic, distributed representations emerging from situated and embodied learning in the environment. Most current neurorobotics studies are based on connectionist-type neural architectures, as multi-layer perceptrons and selforganising maps. For example, many evolutionary robotics models use perceptron-like control architectures to evolve connection weights via genetic algorithms. In the ERA developmental architecture discussed above, the robot's control is based on an ensemble of self-organising maps connected via Hebbian learning. In all these connectionist neurorobotics models, the designer only intends to use neural control architecture to model paralleled and distributed processing strategies in cognitive control and to implement learning via connectionist learning rules, but not to model a realistic implementation of neural areas and pathways involved in motor control.

More recently, a few neuro-robotics models have used more realistic implementations of brain-like circuitry and neurons, to take direct inspiration from neuroscience findings on the distributed control of behaviour. These models range from the implementation of the different brain areas and pathways involved in behaviour control (though still using connectionist, functional level implementation of neuron activations and learning rules), to a more realistic implementation of individual neurons (e.g. Spiking neurons) and learning mechanisms. These for example, include the use of Spike Timing Dependent Plasticity (STDP) that implement associative learning. In the computational embodied neurorobotics approach of the TROPICALS model [41], a series of Kohonen maps and population code maps are devoted to model specific brain areas. In this model, modules exist for the pre-motor cortex (PMC), early visual areas (V1-V2) and the pre-frontal cortex (PFC). This architecture is used to control a simulated iCub to learn the affordance of objects, to model compatibility effects and to perform mental rotation tasks [42].Neurorobotics studies aiming at an even more realistic implementation of the robot's neurocontrol system use models of realistic biological neurons and learning rules. These typically utilize Spiking Neural Networks [43] (SNN), i.e. networks of neurons capable of producing spikes of activities following changes in the membrane potential. Learning is implemented with rules such as the STDP, which models a temporally asymmetric Hebbian rule established on time-based correlation between pairs of connected neurons. Examples of SNN robotics models are reaching prototype of Joshi \& Maass [44] and Bouganis \& Shanahan [45] and operant conditioning learning prototype developed by Helgadottir [52].

The use of SNN for robots has been further supported by the availability of neuromorphic systems, i.e. novel architectures which implements computational neuroscience models directly into the hardware. Amongst these, the SpiNNaker neuromorphic system has been specifically designed for implementation of spiking neurons. This is a universal neural network platform designed for real-time simulation with an array of programmable cores operating in parallel over a configurable asynchronous multicast interconnect, that can be easily programmed by users with a wide range of different models [46]. The SpiNNaker architecture has been integrated with a SNN model of attention to control goal-directed selective attention in the humanoid robot iCub [47]. The behavioral model was based on Galluppi et al. [48] SNN model of goaldirected selective attention for two objects (one vertical and one horizontal, of which one is always preferred and thus must be looked at).The SNN model takes as input, via a retina layer, an image of two objects, via the robot's camera. The camera image is down sampled to a $16 \times 16$ image of black and white pixels, which are then converted to spikes by mapping 'ON' pixels to spike outputs (See Fig. 5a/5b). The neural architecture (Fig.6) simulates four interconnected brain areas involved in visual attention: three visual cortex layers (V1, V2, V4), the prefrontal cortex layer (PFC) driving attention towards the (prewired) preferred object orientation, and a winner-take-all 
Lateral Intraparietal cortex (LIP) area, involved for the attention of topographic selection and location, which will control the robot's gaze behavior (in humans, the LIP area is involved in gaze, especially in saccadic movements and in eyes tracking movements).

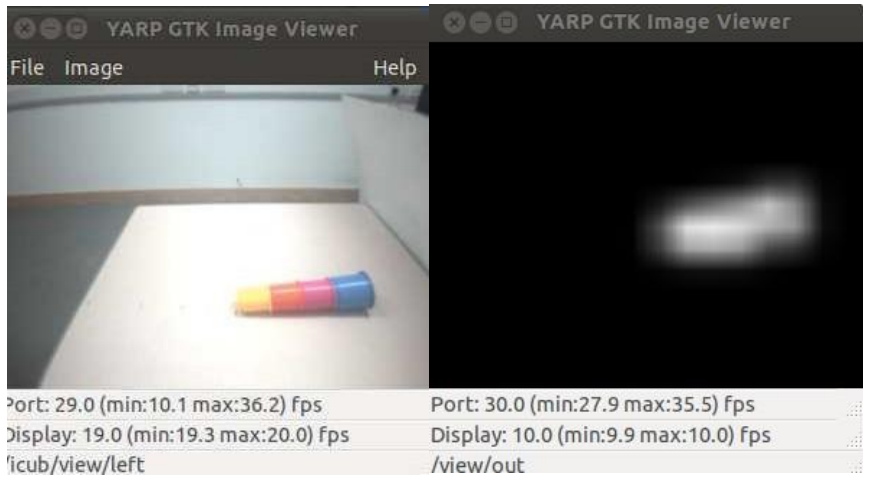

Fig.5 a) Robot's camera view.

b) Effect of preprocessing.

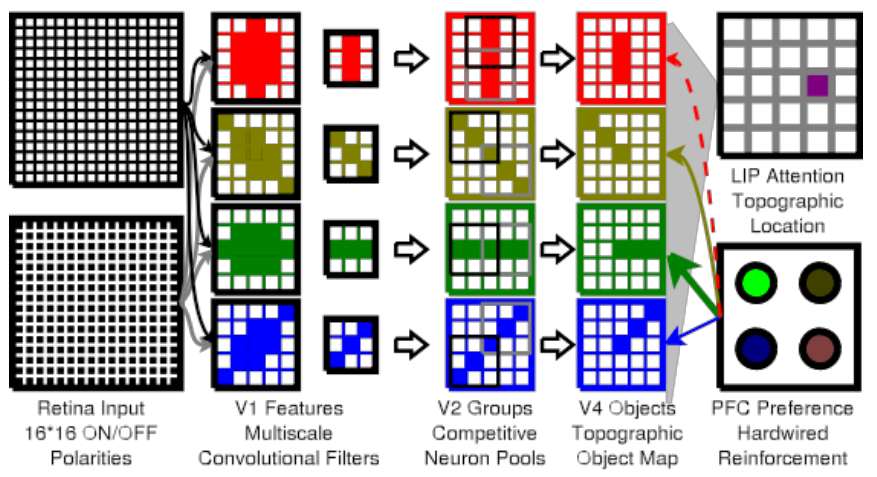

Fig.6 Architecture of the Spiking Neural Networks model: Retina Input, V1, V2, V4, LIP and PFC.

A set of experiments were carried out to test the ability of neural architecture to control selective attention for the iCub robots and its capability for localizing and attending to the preferred object. In one experiment, the robot is tested with a single object, whilst in the second experiment two objects are shown. Results for example show that in 20 runs of the network with different parameters, 14 resulted in the correct object being selected.

This model has been further extended to add a language learning component. Taking inspiration from a neuro-anatomical model of visuo-motor cell assemblies, the attention model was extended by adding an additional layer for the auditory cortex. This new layer receives as input speech signals, which can for example correspond to the name of the object and/or its properties (e.g., color). The extended architecture has been in fact tested for object naming experiments, with the use of the STDP rule for the learning of vision-speech association [47].

The neurorobotics approach, and specifically the latest developments in the integration of the robot platforms with neuromorphic systems, offers a novel approach to the design of fully autonomous systems. In addition to the design of robots capable of learning using brain-like control systems, other advantages of systems like SpiNNaker are, for example, efficiency in energy consumption, which is a key factor in the design of fully autonomous robots.

\section{BCI INTERFACING ROBOTICS}

The common thread in the discourse addressed in the previous sections, inevitably leads to a short to discuss and clarify the results obtained through the connection of Brain Computer Interface with robotic systems, especially in assistive robotics[49]. The recent reviews [50] [51] already indicated the existence of products that interface BCI systems with robotic environments, especially in the field of entertainment (e.g., Neural Impulse Actuator, Star Wars Force Training, Mind Flex). One of the early works in this regard has been permeated with the objective of mechanically rebuilding prehensile capacity, through an analysis of the EEG frequencies that are implied in 'grasping activities'. The primary motor cortex, prefrontal, sensory-motor cortex and visual-motor cortex are involved in grasping movements, but the neural characterization at cortical EEG activities, is poorly coded in these areas. Therefore, the decoding of the kinematics of grasping has aroused great interest, especially for the construction of a Brain Machine Interface which could extrapolate the intention of movement and that could interface and control in such fashion external device, especially humanoid robotics systems[52]. In this way, humanoids robots can become an active user's biofeedback. However, such process is not simple because the kinematic space, given by the joint angle and the speed of the movement synergies, is encrypted by potential cortical fields. For example, in Agashe's work (described in section II) the subjects had to grasp various objects, during a BCI recording, with their natural right hand and through a robotic glove that specifically caught the trajectories by 18 joint angles.

Other recent works, like Chae's research, describe humanoid robots actuating by BCI [53]. These studies developed humanoid robotics systems which are able to be guided through mind activity (EEG rhythms). These systems provided lowlevel motion commands (e.g. right, left, forward) by combining the classification of three motor imagery conditions (i.e., right hand motion, left hand motion and foot motion). A similar study on hybrid robotics system [54] describes a low cost interface that allows users to control navigation and space investigation. This process is actuated by a humanoid robot that is able to recognize the chosen object, by following $\mathrm{BCI}$ signals based on SSVEP and ERD/ERS

Therefore, it remains to be verified whether such a hybrid approach can be adapted to persons with severe disabilities, who are in condition to imagine the motor gesture. In particular, the latest research suggests that the imagery and motor training allows to restore the control of neural areas that are able to perform prehensile tasks [55], required to interface robotics prosthesis [56]. According to this prospective, Chella's research[57], is dedicated to patients with Amyotrophic Lateral Sclerosis and others neuromuscular diseases. In this interesting work, Chella et al., developed a museum robotics guide interfaced with a BCI system. These innovative studies 
demonstrate the possibility that a subject can control a robot, by synchronizing mentally in a human-machine interaction system. Such results therefore highlight, as described, that hybrid interfaces are highly suitable for people with physical disabilities: e.g., from the motor control of a wheelchair, to the control of a humanoid robotic system, to assistive robotic systems which are also useful in domotics solutions.

\section{CONCLUSION}

This review, in addition to introducing the latest technologies developed within the fields of human-robot interaction and neuroprosthetics, shows that highly interdisciplinary approaches, such as those related to perceptron studies, computational neuroscience, robotics and cognitive science, converge towards the building and re-modulation of a functional process. In this human-robot interaction, of particular relevance, is the concept of proxemic space[58], which is the personal space that people keep around themselves and which becomes a cognitive and behavioural extension of the subject. Here the function of space and the use of proxemics through technology [59] becomes an extension of the user. So, the users, via such interaction technologies, can enhance their effector system, adapting it to the 'central system' person. This happens without the need to ever define an end to remodeling and learning feedback between the human system and the effector or robotic system. Furthermore, this interaction, between cognitive neuroscience, engineering, robotic systems and neuroprosthetics becomes highly valid, not only for functional applications, but also on theoretical applications which allow the understanding of neural modules. That is, through the construction of neural interfaces, it also becomes possible to obtain insight into the physiological neural functioning too. The development of adaptive and learning systems, as with the humanoid robotics models reviewed above, has important implications to the overcoming of the current limitations preventing robots from working with humans. For example, current industrial assembly robots are rigidly preprogrammed to perform a fixed set of repetitive actions, requiring a clear, physical barrier between the robot's workspace and the human's workspace. On the contrary, robots which are capable to learn from interaction with humans, e.g. via action imitation or linguistic instructions, can dynamically adapt their behaviour to the safety and collaborative requirements of the human operator. These learning capabilities, associated with the latest developments in soft materials, is also leading to the design of compliant robots, i.e. robotic platforms that can safely share workspace with humans because their soft mechanics (e.g. elastic or spring actuators) prevent harm to the human users [60]

Within industrial and commercial process, especially in the electronics and bionics field, the adaptive interaction between humans, prosthetics and robotic systems is becoming more and more important. This sets the bases for research facilities, educational institutions and industrial research and development bodies are joining forces for the development of innovative human-machine interaction systems.

\section{REFERENCES}

[1] M. A. Lebedev and M. A. L. Nicolelis, "Toward a whole-body neuroprosthetic," Prog. Brain Res., vol. 194, pp. 47-60, 2011.

[2] J. Schmidhuber, "Deep Learning in neural networks: An overview," Neural Networks, vol. 61. Elsevier Ltd, pp. 85-117, 2015.

[3] A. Krizhevsky, I. Sutskever, and G. E. Hinton, "ImageNet Classification with Deep Convolutional Neural Networks," $A d v$. Neural Inf. Process. Syst., pp. 1-9, 2012.

[4] P. Dario, P. F. M. J. Verschure, T. Prescott, G. Cheng, G. Sandini, R. Cingolani, R. Dillmann, D. Floreano, C. Leroux, S. MacNeil, P. Roelfsema, X. Verykios, A. Bicchi, C. Melhuish, and A. AlbuSch??ffer, "Robot companions for citizens," in Procedia Computer Science, 2011, vol. 7, pp. 47-51.

[5] K. . Dautenhahn, S. . Woods, C. . Kaouri, M. L. . Walters, K. L. Koay, and I. Werry, "What is a robot companion-friend, assistant or butler?," in Intelligent Robots and Systems, 2005, vol. IEEE/RSJ I, pp. $1192-1197$.

[6] J. Kennedy, P. Baxter, and T. Belpaeme, "The Robot Who Tried Too Hard: Social Behaviour of a Robot Tutor Can Negatively Affect Child Learning," in Proceedings of the Tenth Annual ACM/IEEE International Conference on Human-Robot Interaction, 2015, pp. 67-74.

[7] J. R. Wolpaw, D. J. McFarland, and T. M. Vaughan, "Braincomputer interface research at the Wadsworth Center," IEEE Trans. Rehabil. Eng., vol. 8, no. 2, pp. 222-226, 2000.

[8] B. Z. Allison, D. J. McFarland, G. Schalk, S. D. Zheng, M. M. Jackson, and J. R. Wolpaw, "Towards an independent braincomputer interface using steady state visual evoked potentials.," Clin. Neurophysiol., vol. 119, no. 2, pp. 399-408, 2008.

[9] J. J. Vidal, "Vidal.pdf," Annu. Rev. Biophys. Bioeng., vol. 2, pp. $157-180,1973$

[10] M. M. Jackson and R. Mappus, "Brain-Computer Interfaces," pp. 21-34, 2010.

[11] C. Guger, C. Kapeller, R. Ortner, and K. Kamada, "Motor Imagery with Brain- Computer Interface Neurotechnology," 2015.

[12] B. Blankertz, F. Losch, M. Krauledat, G. Dornhege, G. Curio, and K. R. M??ller, "The Berlin brain - computer interface: Accurate performance from first-session in BCI-na??ve subjects," IEEE Trans. Biomed. Eng., vol. 55, no. 10, pp. 2452-2462, 2008.

[13] Neurosky, "Neurosky Mindwave," www.neurosky.com. 2014.

[14] J. Katona, I. Farkas, T. Ujbanyi, P. Dukan, and A. Kovari, "Evaluation of the NeuroSky MindFlex EEG headset brain waves data," in SAMI 2014 - IEEE 12th International Symposium on Applied Machine Intelligence and Informatics, Proceedings, 2014, pp. 91-94.

[15] S. Invitto, C. Faggiano, S. Sammarco, V. De Luca, L. T. De Paolis, F. Lamberti, A. Sanna, and J. Rokne, "Haptic, Virtual Interaction and Motor Imagery: Entertainment Tools and Psychophysiological Testing," 2016.

[16] V. Gallese and C. Sinigaglia, "What is so special about embodied simulation?," Trends Cogn. Sci., vol. 15, no. 11, pp. 512-519, 2011.

[17] S. Hurley, T. Shared, C. Model, H. Control, S. C. Enable, and S. Hurley, "Editorial Note: The shared circuits model ( SCM ): How control , mirroring, and simulation can enable imitation , deliberation , and mindreading," pp. 1-58, 2008.

[18] M. J. Pearson, a. G. Pipe, C. Melhuish, B. Mitchinson, and T. J. Prescott, "Whiskerbot: A robotic active touch system modeled on the rat whisker sensory system," Adapt. Behav., vol. 15, no. 3, pp. 223-240, 2007.

[19] I. B. Olivier and Poupyrev, "REVEL: Tactile Feedback Technology for Augmented Reality," ACM Trans. Graph., vol. 31, no. 4, pp. 111, 2012.

[20] B. Matulevich, V. Pandit, C. Lin, G. E. Loeb, and J. A. Fishel, "LOW-COST, COMPLIANT CONTACT SENSOR FOR FRAGILE GRASPING WITH REDUCED COGNITIVE LOAD," pp. 2-5.

[21] J. A. Fishel, S. Member, G. E. Loeb, and S. Member, "Sensing Tactile Microvibrations with the BioTac - Comparison with Human Sensitivity," pp. 1122-1127, 2012.

[22] U. B. Rongala, A. Mazzoni, and C. M. Oddo, "Neuromorphic artificial touch for categorization of naturalistic textures," IEEE Trans. NEURAL NETWORKS Learn. Syst., pp. 1-11, 2015.

[23] H. A. Agashe, A. Y. Paek, Y. Zhang, and E. W. Sellers, "Global 
cortical activity predicts shape of hand during grasping," vol. 9, no. April, pp. 1-11, 2015.

[24] G. Pfurtscheller, B. Z. Allison, C. Brunner, G. Bauernfeind, T. Solis-Escalante, R. Scherer, T. O. Zander, G. Mueller-Putz, C. Neuper, and N. Birbaumer, "The hybrid BCI.," Front. Neurosci., vol. 4, p. 30, 2010.

[25] D. Vernon, Artificial Cognitive Systems, no. October. Cambridge MIT press, 2014.

[26] S. Nolfi and D. Floreano, "Synthesis of autonomous robots through evolution," Trends in Cognitive Sciences, vol. 6, no. 1. pp. 31-37, 2002.

[27] M. Cangelosi, A; Schlesinger, Developmental Robotics: From Babies to Robot. 2015.

[28] J. L. Krichmar, A. K. Seth, D. A. Nitz, J. G. Fleischer, and G. M. Edelman, "Spatial navigation and causal analysis in a brain-based device modeling cortical-hippocampal interactions.," Neuroinformatics, vol. 3, no. 3, pp. 197-221, 2005.

[29] A. Cangelosi, G. Metta, G. Sagerer, S. Nolfi, C. Nehaniv, K. Fischer, J. Tani, T. Belpaeme, G. Sandini, F. Nori, L. Fadiga, B. Wrede, K. Rohlfing, E. Tuci, K. Dautenhahn, J. Saunders, and A. Zeschel, "Integration of action and language knowledge: A roadmap for developmental robotics," IEEE Trans. Auton. Ment. Dev., vol. 2, no. 3, pp. 167-195, 2010.

[30] R. Pfeifer and J. C. Bongard, How the Body Shapes the Way We Think: A New View of Intelligence. 2006.

[31] W. J. Clancey, Situated cognition: on human knowledge and computer representations. Cambridge University Press, 1997.

[32] L. W. Barsalou, "Grounded cognition.," Annu. Rev. Psychol., vol. 59, pp. 617-645, 2008.

[33] G. Pezzulo, L. W. Barsalou, A. Cangelosi, M. H. Fischer, K. McRae, and M. J. Spivey, "Computational grounded cognition: A new alliance between grounded cognition and computational modeling," Frontiers in Psychology, vol. 3, no. JAN. 2013.

[34] L. K. Samuelson, L. B. Smith, L. K. Perry, and J. P. Spencer, "Grounding word learning in space," PLoS One, vol. 6, no. 12, 2011.

[35] A. F. Morse, V. L. Benitez, T. Belpaeme, A. Cangelosi, and L. B. Smith, "Posture affects how robots and infants map words to objects," PLoS One, vol. 10, no. 3, 2015.

[36] A. Eccleston, N. DeWitt, C. Gunter, B. Marte, and D. Nath, "Epigenetics," Nature, vol. 447, no. 7143, pp. 395-395, 2007.

[37] S. Song, K. D. Miller, and L. F. Abbott, "Competitive Hebbian learning through spike-timing-dependent synaptic plasticity.," Nat. Neurosci., vol. 3, no. 9, pp. 919-926, 2000.

[38] B. Anderson, "Kohonen neural networks and language.," Brain Lang., vol. 70, no. 1, pp. 86-94, 1999.

[39] F. Stramandinoli, D. Marocco, and A. Cangelosi, "The grounding of higher order concepts in action and language: A cognitive robotics model," Neural Networks, vol. 32, pp. 165-173, 2012.

[40] M. Ruciński, A. Cangelosi, and T. Belpaeme," "Robotic model of the contribution of gesture to learning to count," 2012 IEEE Int. Conf. Dev. Learn. Epigenetic Robot. ICDL 2012, pp. 1-6, 2012.

[41] D. Caligiore, A. M. Borghi, D. Parisi, R. Ellis, A. Cangelosi, and G. Baldassarre, "How affordances associated with a distractor object affect compatibility effects: A study with the computational model TRoPICALS," Psychol. Res., vol. 77, no. 1, pp. 7-19, 2013.

[42] K. Seepanomwan, D. Caligiore, A. Cangelosi, and G. Baldassarre, "Generalisation, decision making, and embodiment effects in mental rotation: A neurorobotic architecture tested with a humanoid robot," Neural Networks, Elsevier Ltd, 2015.

[43] W. Gerstner and W. M. Kistler, "Spiking Neuron Models: Single Neurons, Populations, Plasticity," Book, p. 494, 2002.

[44] P. Joshi and W. Maass, "Movement generation with circuits of spiking neurons.," Neural Comput., vol. 17, no. 8, pp. 1715-38, 2005.

[45] A. Bouganis and M. Shanahan, "Training a spiking neural network to control a 4-DoF robotic arm based on spike timing-dependent plasticity," in Proceedings of the International Joint Conference on Neural Networks, 2010.

[46] S. B. Furber, D. R. Lester, L. A. Plana, J. D. Garside, E. Painkras, S. Temple, and A. D. Brown, "Overview of the SpiNNaker system architecture," IEEE Transactions on Computers, vol. 62, no. 12. pp.
2454-2467, 2013.

[47] A. Cichocki, M. Jankovic, R. Zdunek, and S. Amari, "Neural Information Processing," vol. 4984, pp. 781-790, 2008.

[48] F. Galluppi, K. Brohan, S. Davidson, T. Serrano-Gotarredona, J. P. Carrasco, B. Linares- Barranco, and S. Furber, "A Real-Time, Event-Driven Neuromorphic System for Goal- Directed Attentional Selection," in 19th Int'l. Conf. on Neural Information Processing (ICONIP 2012)., 2012, pp. 226-233.

[49] V. Gandhi, Brain-Computer Interfacing for Assistive Robotics. Elsevier, 2015.

[50] B. Zhang, J. Wang, and T. Fuhlbrigge, "A review of the commercial brain-computer interface technology from perspective of industrial robotics," in 2010 IEEE International Conference on Automation and Logistics, ICAL 2010, 2010, pp. 379-384.

[51] T. Dahl and M. Boulos, "Robots in Health and Social Care: A Complementary Technology to Home Care and Telehealthcare?," Robotics, vol. 3, no. 1, pp. 1-21, 2013.

[52] C. J. Bell, P. Shenoy, R. Chalodhorn, and R. P. Rao, "Control of a humanoid robot by a noninvasive brain-computer interface in humans," J. Neural Eng., vol. 5, no. 2 PG - 214-220, pp. 214-220, 2008.

[53] Y. Chae, J. Jeong, and S. Jo, "Toward brain-actuated humanoid robots: Asynchronous direct control using an EEG-Based BCI," IEEE Trans. Robot., vol. 28, no. 5, pp. 1131-1144, 2012.

[54] B. Choi and S. Jo, "A Low-Cost EEG System-Based Hybrid BrainComputer Interface for Humanoid Robot Navigation and Recognition," PLoS One, vol. 8, no. 9, 2013.

[55] B. Wodlinger, J. E. Downey, E. C. Tyler-Kabara, a B. Schwartz, M. L. Boninger, and J. L. Collinger, "10 dimensional anthropomorphic arm control in a human brain-machine interface: Difficulties, solutions, and limitations," J Neural Eng, vol. In Press, no. 1, p. 016011, 2014.

[56] L. R. Hochberg, D. Bacher, B. Jarosiewicz, N. Y. Masse, J. D. Simeral, J. Vogel, S. Haddadin, J. Liu, S. S. Cash, P. van der Smagt, and J. P. Donoghue, "Reach and grasp by people with tetraplegia using a neurally controlled robotic arm," Nature, vol. 485, no. 7398 , pp. 372-375, 2012.

[57] A. Chella, E. Pagello, E. Menegatti, R. Sorbello, S. M. Anzalone, F. Cinquegrani, L. Tonin, F. Piccione, K. Prifitis, C. Blanda, E. Buttita, and E. Tranchina, "A bci teleoperated museum robotic guide," Proc. Int. Conf. Complex, Intell. Softw. Intensive Syst. CISIS 2009, pp. 783-788, 2009.

[58] O. M. M. Watson and T. D. D. Graves, "Quantitative Research in Proxemic Behavior," Am. Anthropol., vol. 68, no. 4, pp. 971-985, 1966.

[59] L. Takayama and C. Pantofaru, "Influences on proxemic behaviors in human-robot interaction," 2009 IEEE/RSJ Int. Conf. Intell. Robot. Syst. IROS 2009, pp. 5495-5502, 2009.

[60] A. Albu-Schaffer, O. Eiberger, M. Grebenstein, S. Haddadin, C. Ott, T. Wimböck, S. Wolf, and G. Hirzinger, "Soft robotics," IEEE Robot. Autom. Mag., vol. 15, no. 3, pp. 20-30, 2008. 